\title{
Rabdomiomas cardiacos múltiples, diagnóstico prenatal. Reporte de casos
}

\author{
Multiple cardiac rhabdomyomas, prenatal diagnosis. Case report
}

Tania Carrillo-Lima ${ }^{*}$, Sandra L. Pacheco-López ${ }^{1}$, Paola L. Castro-Santiago ${ }^{1}$ y Luis F. Oseguera-Torres²

${ }^{1}$ Departamento de Cardiología Pediátrica, Centro Médico Nacional de Occidente, Instituto Mexicano del Seguro Social, Unidad Médica de Alta Especialidad Hospital de Pediatría; ${ }^{2}$ Departamento de Medicina Fetal, Centro Médico Nacional de Occidente, Instituto Mexicano del Seguro Social, Unidad Médica de Alta Especialidad Hospital de Gineco Obstetricia. Guadalajara, Jal., México

\section{Resumen}

Los tumores cardiacos son raros en la población infantil, teniendo una incidencia máxima reportada del 0.027\% en el diagnóstico prenatal, incrementándose la incidencia en el diagnóstico por necropsia. Los rabdomiomas son los tumores cardiacos más frecuentes, algunos casos pueden asociarse con esclerosis tuberosa. Presentamos el reporte de dos casos en nuestra unidad a los cuales se les realizó diagnóstico prenatal con seguimiento posterior al nacimiento y asociándose uno de ellos a esclerosis tuberosa. Ambos casos recibieron atención en un centro de tercer nivel, sin complicaciones, sin requerir tratamiento quirúrgico, pudiendo ser egresados.

Palabras clave: Rabdomiomas. Esclerosis tuberosa. Diagnóstico prenatal.

\begin{abstract}
Cardiac tumors are rare in children, having a maximum reported incidence of $0.027 \%$ in prenatal diagnosis, increasing the incidence in necropsy diagnosis; rhabdomyomas are the most frequent cardiac tumors, some cases are associated with tuberous sclerosis. We present the report of two cases in our unit that were diagnosed prenatal with follow-up after birth and one of them was associated with tuberous sclerosis. Both cases were admitted in a third level center, uncomplicated, without requiring surgical treatment, and could be discharged.
\end{abstract}

Keywords: Rhabdomyomas. Tuberous sclerosis. Prenatal diagnosis.

\section{Introducción}

Los tumores cardiacos primarios son raros en todos los grupos de edad, con una incidencia del 0.0017$0.027 \%$, en la necropsia en población infantil se informa que la incidencia es del $0.25 \%{ }^{1}$. Los rabdomiomas son los tumores cardiacos más frecuentes en niños, seguidos de fibromas (25 a 30\%) y con menos frecuencia mixomas, lipomas, teratomas, hemangiomas, mesoteliomas y tumores de células de Purkinje. Suelen asociarse a esclerosis tuberosa, el cual puede afectar

\section{Correspondencia:}

*Tania Carrillo-Lima

E-mail: taniac.lima7@gmail.com
Disponible en internet: 23-02-2021

Arch Cardiol Mex. 2022;92(1):94-98 www.archivoscardiologia.com

1405-9940 / @ 2021 Instituto Nacional de Cardiología Ignacio Chávez. Publicado por Permanyer. Este es un artículo open access bajo la licencia CC BY-NC-ND (http://creativecommons.org/licenses/by-nc-nd/4.0/). 
a todos los órganos del cuerpo, siendo más común cerebro, riñón, corazón y pulmones².

Presentamos dos casos clínicos de rabdomiomas cardiacos diagnosticados en nuestra unidad, haciendo énfasis en el resultado a nivel cardiaco y su asociación con esclerosis tuberosa.

\section{Material y métodos}

Por referencia a nuestra unidad de alta especialidad, todas las mujeres con embarazo de alto riesgo son monitoreadas con ecocardiografía fetal. Durante los años 2018 y 2019 se documentaron dos casos de rabdomiomas intracardiacos. Los exámenes se realizaron en el tercer trimestre del embarazo, excluyendo los casos de cardiopatías congénitas.

El ecocardiograma prenatal se realizó transabdominal, con sistema Philips IE33 con transductor sectorial 8S. Se adquirieron imágenes en modo bidimensional, modo M y Doppler pulsado y continuo. El diagnóstico se confirmó realizándose ecocardiogramas posnatales en el mismo tipo de equipo, incluyéndose un examen clínico y electrocardiograma. Con seguimiento posterior a la consulta externa de cardiología pediátrica.

\section{Reporte de casos}

- El primer caso se trata de una paciente de 20 años de edad, sin antecedentes heredofamiliares de importancia, la cual inicia control de embarazo de alto riesgo en tercer nivel por múltiples amenazas de aborto. Derivada a nuestro hospital a las 36 semanas de gestación, realizándose el primer ecocardiograma prenatal por parte de nuestro servicio detectando tumores cardiacos compatibles con rabdomiomas, con imagen ecogénica en aurícula derecha de 18 $\mathrm{mm}$ y en ventrículo derecho de $20 \mathrm{~mm}$ con septum interventricular de $8 \mathrm{~mm}$ (Fig. 1), sin datos de obstrucción al tracto de salida, por lo que se decide continuar con el embarazo. Nace por cesárea, sin complicaciones. Se realiza ecocardiograma posnatal de control con los hallazgos previos, reportando tumoración a nivel de la cava superior de $13 \times 18 \mathrm{~mm}$, tumoración en surco atrioventricular de $15.4 \mathrm{x}$ $4.5 \mathrm{~mm}$, en ventrículo derecho con tumoración de $17.4 \times 22 \mathrm{~mm}$, siendo bilobulado, a nivel del ventrículo izquierdo con múltiples tumoraciones adheridas al septum y a la pared anterior la mayor de $7.7 \mathrm{x}$ $8.2 \mathrm{~mm}$ (Fig. 2), corroboradas por resonancia magnética (Fig. 3). Debido a la asociación de tumores intracardiacos con esclerosis tuberosa fue valorado

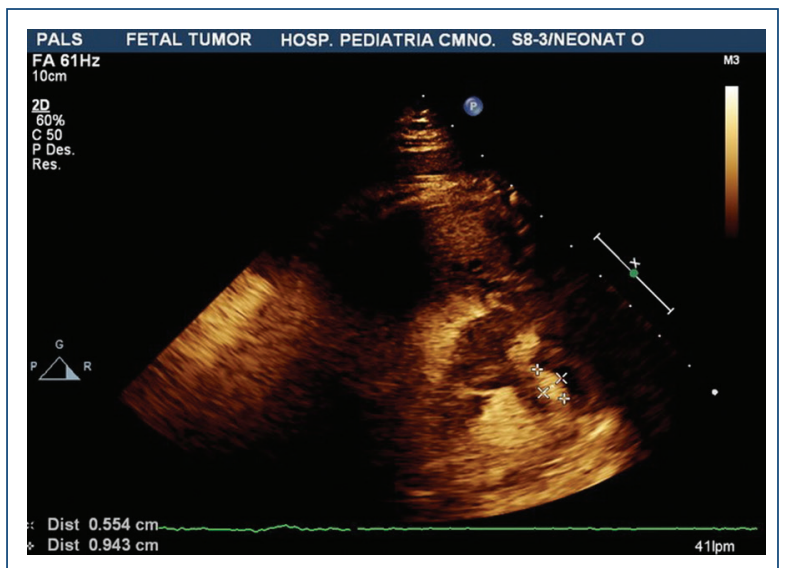

Figura 1. Imagen del ecocardiograma fetal donde se observa en cuatro cámaras $2 \mathrm{D}$ imagen de tumor en ventrículo derecho de $20 \mathrm{~mm}$ con septum interventricular de $8 \mathrm{~mm}$.

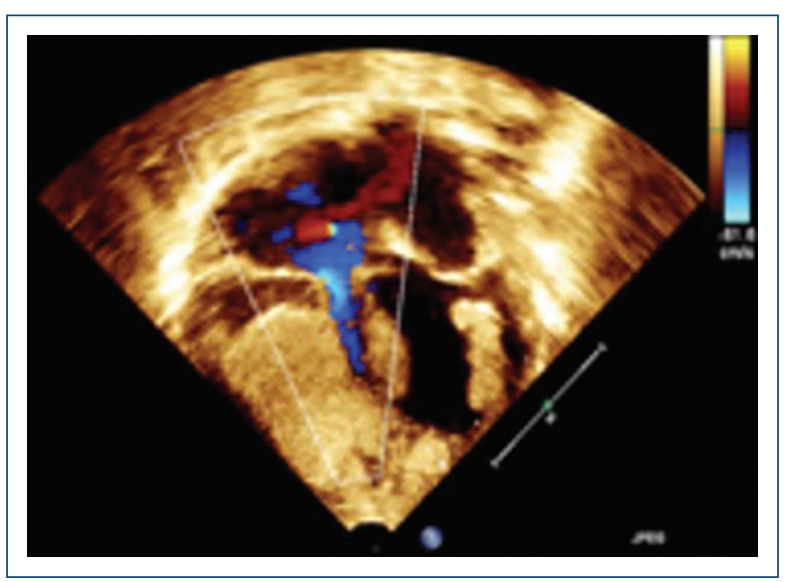

Figura 2. Imagen del ecocardiograma posnatal del mismo paciente, donde se observa en cuatro cámaras $2 \mathrm{D}$ tumor en surco atrioventricular de $15.4 \times 4.5 \mathrm{~mm}$, en ventrículo derecho con tumor de $17.4 \times 22 \mathrm{~mm}$ y a nivel del ventrículo izquierdo con múltiples tumores adheridos al septum y a la pared anterior la mayor de $7.7 \times 8.2 \mathrm{~mm}$.

por el Servicio de Neurología, en donde mediante la realización de resonancia magnética de encéfalo se evidencia lesión nodular intraaxial en el giro recto derecho y nódulos subependimarios en ambos ventrículos laterales (Fig. 4), con hallazgos referidos que se encuentran dentro del espectro de esclerosis tuberosa. Se mantiene en vigilancia, en control por la consulta externa de dicho servicio.

Valorado por el Servicio de Electrofisiología por el riesgo de desarrollo de arritmia, como parte de protocolo de estudio, con electrocardiograma y Holter de 24 horas, encontrando frecuencia cardiaca media 


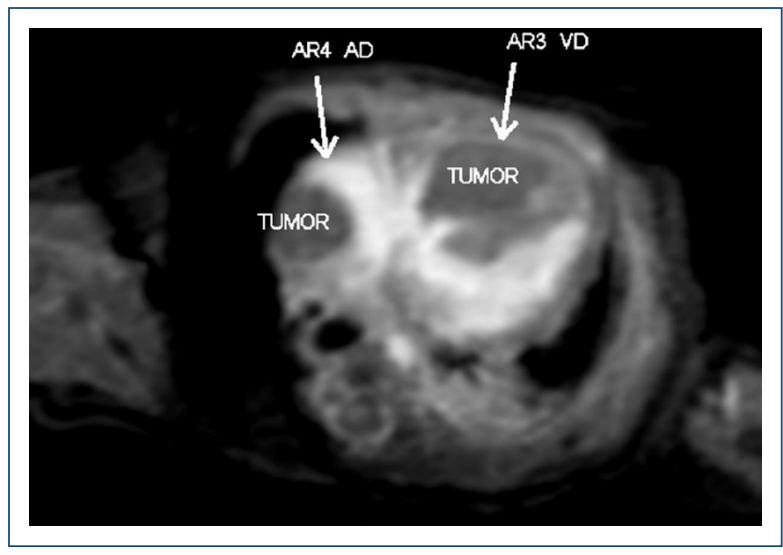

Figura 3. Imagen posnatal de la resonancia magnética en secuencia de sangre blanca, eje cuatro cámaras, donde se observan imágenes isointensas en aurícula y ventrículo derechos, característica de rabdomiomas.

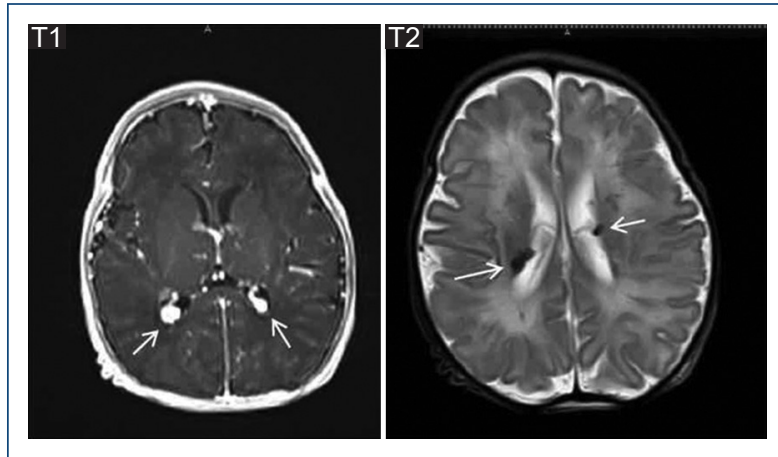

Figura 4. Resonancia magnética de encéfalo, donde se observan múltiples lesiones nodulares periventriculares y subependimarias bilaterales con una intensidad de señal predominantemente alta en T1 y baja a intermedia en T2.

de 156 lpm, frecuencia máxima de $250 \mathrm{lpm}$. El total de QRS registradas fue de 166,470 , lo cual representa el $11.8 \%$ del total de QRS registradas, iniciando tratamiento con propranolol, en seguimiento por la consulta externa.

- Nuestro segundo caso corresponde a una paciente de 26 años de edad, sin antecedentes heredofamiliares de importancia, la cual inicia control de embarazo de alto riesgo en tercer nivel por hallazgo en ultrasonido fetal de control imágenes sugestivas de tumores intracardiacos. Derivada a nuestro hospital a las 35 semanas de gestación, se realizó el primer ecocardiograma por parte de nuestro servicio en este periodo, en el cual se detectó imagen hiperecogénica a nivel del aparato valvular izquierdo de $11 \times 7 \mathrm{~mm}$, la cual impresiona

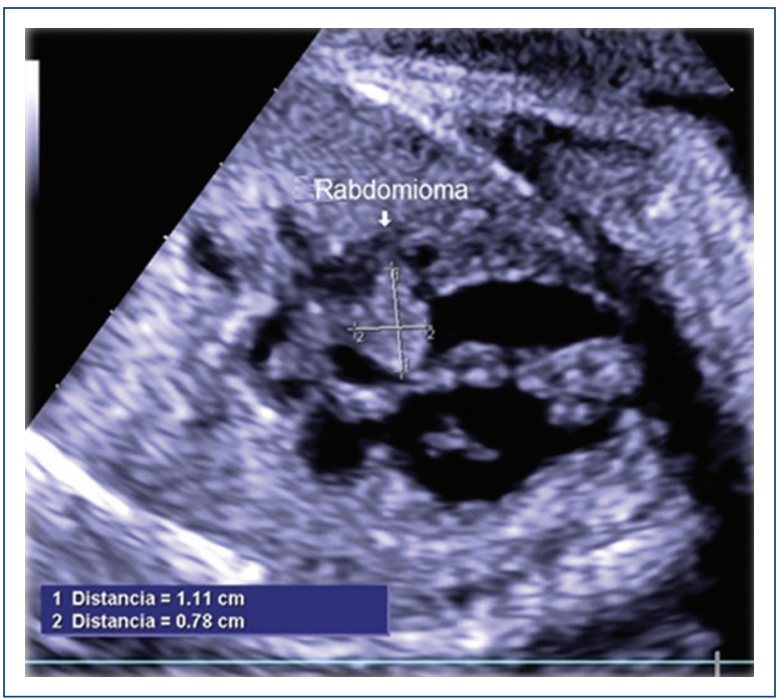

Figura 5. Imagen del ecocardiograma fetal donde se observa en eje largo 2D tumor a nivel del aparato valvular izquierdo de $11 \times 7 \mathrm{~mm}$, además de otra imagen de mismas características en pared externa de ventrículo izquierdo de $7 \times 7 \mathrm{~cm}$.

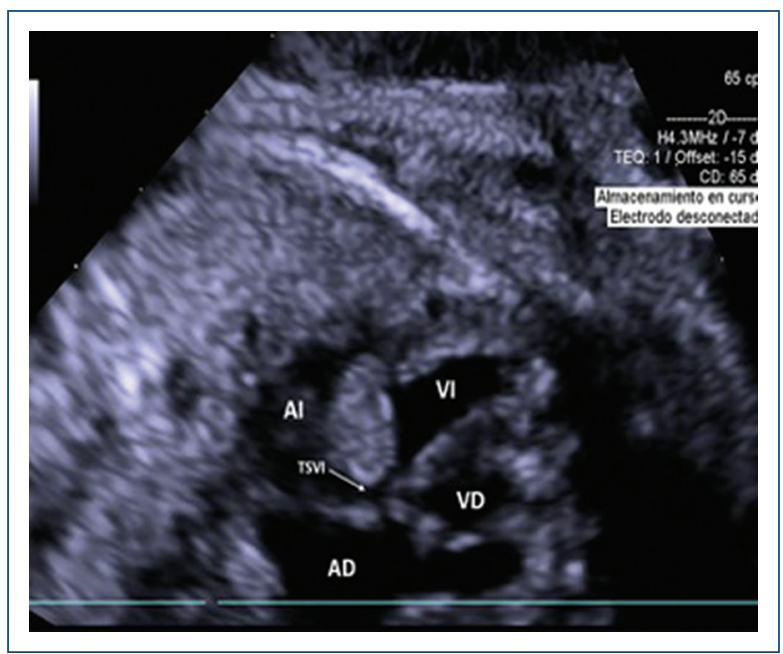

Figura 6. Imagen del ecocardiograma fetal donde se observa en cinco cámaras $2 \mathrm{D}$ el mismo tumor a nivel del aparato valvular izquierdo, el cual impresiona compresión a nivel del tracto de salida del ventrículo izquierdo.

compresión de la válvula aórtica; se observa otra imagen hiperecogénica de $8 \times 6 \mathrm{~mm}$ en pared externa de ventrículo derecho, adyacente a válvula tricúspide se observa otra imagen de las mismas características en pared externa de ventrículo izquierdo de $7 \times 7 \mathrm{~cm}$ (Figs. 5 y 6), sin datos de obstrucción al tracto de salida, por lo que se decide continuar con el embarazo. Nace por cesárea programada, sin complicaciones. 
Valorado por el Servicio de Electrofisiología con electrocardiograma y Holter de 24 horas, sin alteraciones en ritmo, por lo que se mantiene solo en vigilancia por la consulta externa.

En este caso también se realizó pesquisa para descartar esclerosis tuberosa, descartándose por estudio de imagen cerebral.

\section{Discusión}

Los tumores cardiacos son extremadamente raros en niños $(0.027$ a $0.17 \%)$. La gran mayoría de los tumores cardiacos primarios en los niños son benignos, mientras que aproximadamente el $10 \%$ son malignos ${ }^{2}$. De este total, los rabdomiomas son los tumores cardiacos más comunes, presentándose en el $43-60 \%$ de los casos, y se encuentran asociados a la esclerosis tuberosa ${ }^{3}$.

Esta es una condición dominante autosómica con penetrancia y expresividad variables, aunque la mayoría de los casos nuevos representan nuevas mutaciones. Se han identificado al menos dos locus genéticos, en el brazo largo del cromosoma 9 (9q34) y en el brazo largo del cromosoma 11 (11q14.1-23). Se desconoce el déficit genético específico en sí, así como el proceso subyacente por el cual se produce el tumor'.

Los rabdomiomas se diagnostican fácilmente por ultrasonido, debido a su aspecto hiperecogénico, con apariencia de masas nodulares hiperecoicas múltiples ubicadas en las cavidades cardiacas. La mejor exploración para evaluar correctamente la masa del corazón es la vista de cuatro cámaras, la ecocardiografía permite una determinación precisa de la extensión y ubicación de la masa ${ }^{5}$. Aunque los rabdomiomas pueden formarse en cualquier parte del corazón, generalmente se detectan en los ventrículos y las áreas septales interventriculares y a menudo son masas múltiples.

El hallazgo de estos tumores representan un factor de riesgo para insuficiencia cardiaca y muerte súbita neonatal intrauterina, siendo importante el estudio con Doppler, útil para evaluar posibles modificaciones en la hemodinámica intracardiaca ${ }^{6}$.

La presencia de múltiples tumores que afectan al miocardio ventricular es indicativo de rabdomiomas. Cuando se aísla la lesión, se debe realizar un diagnóstico diferencial con respecto a otros tumores cardiacos, como teratomas, fibromas y hemangiomas. Los teratomas son las segundas neoplasias más frecuentes en la infancia ${ }^{7}$. La mayoría de ellos son extracardiacos y se encuentran en la base del corazón. Los fibromas apenas se diferencian de los rabdomiomas debido a su apariencia ecográfica similar; a menudo son pedunculados, pueden crecer hasta tamaños enormes y pueden sufrir degeneración quística y calcificada ${ }^{8}$.

También se debe evaluar el ritmo cardiaco fetal, pues en los raros casos de localización auricular pueden surgir arritmias ${ }^{9}$. El tamaño del tumor debe controlarse durante el embarazo para evaluar el posible crecimiento del tumor, lo que puede deberse a una alta concentración de hormona gestacional que estimula una respuesta hiperplásica e hipertrófica en el tumor ${ }^{10}$.

En un metaanálisis realizado por Chao, et al. estos informaron de que la mayoría de los rabdomiomas cardiacos se detectaron después de la 24. a semana de gestación y solo el $13.7 \%$ de los casos se detectaron antes de la semana 24 de gestación ${ }^{11}$. En nuestros casos, los diagnósticos se realizaron en el último trimestre del embarazo, cerca del término de este, debido a que las pacientes no programaron controles en el primer e inicio del segundo trimestre ${ }^{12}$.

La involución puede estar relacionada con la incapacidad de los tumores para dividirse mientras crecen las cámaras cardiacas y esta consideración puede indicar que algunos factores aún incompletamente identificados, involucrados en la regulación homeostática de la biología cardiaca, podrían conducir a la regresión de las masas ${ }^{13}$.

De hecho, después del nacimiento, las células de rabdomioma pierden su capacidad de dividirse y la regresión del tumor en la infancia es un resultado esperado, independientemente del tamaño del tumor. La resolución completa de más del $80 \%$ de los tumores puede ocurrir durante la primera infancia. La regresión puede dejar una pared de cámara delgada con cicatrices ${ }^{14}$.

En el caso de los rabdomiomas asintomáticos, la cirugía cardiaca es innecesaria, porque los rabdomiomas disminuyen después del nacimiento a la edad de dos meses a dos años con una media de ocho meses, pero deben realizarse visitas pediátricas regulares para detectar un diagnóstico posterior de esclerosis tuberosa. Si el niño se ve afectado por esta patología, se requiere asesoramiento genético de los padres después del nacimiento 0 la interrupción del embarazo ${ }^{15,16}$.

La recomendación actual es un enfoque conservador cuando sea posible, dada la regresión espontánea bien documentada de los rabdomiomas, especialmente para pacientes de menor edad y tumores de menor tamaño, evitándoles la morbilidad de la cirugía ${ }^{17}$. Sin embargo, un estudio reciente mostró que aproximadamente el $25 \%$ de un grupo de pacientes requirió intervención 
quirúrgica y algunos autores abogan por la intervención temprana como una estrategia profiláctica para evitar complicaciones relacionadas con la masa, como embolización tumoral, insuficiencia valvular grave y arritmias ventriculares ${ }^{18}$.

\section{Conclusiones}

Los tumores cardiacos fetales son infrecuentes y aunque regularmente son benignos, su manejo y seguimiento será normado de acuerdo con su evolución clínica. El estudio ecocardiográfico fetal ha permitido un diagnóstico prenatal precoz, con toma de decisiones respecto a la intervención médica o quirúrgica a seguir, mejoría en el control prenatal y posnatal, con tratamiento oportuno de estas, mejorando la supervivencia y el pronóstico de estos pacientes.

\section{Agradecimientos}

A todas las personas involucradas en la realización del protocolo de estudio completo en nuestros pacientes para su documentación adecuada.

\section{Financiamiento}

Ninguno.

\section{Conflicto de intereses}

Los autores declaran no tener conflicto de intereses.

\section{Responsabilidades éticas}

Protección de personas y animales. Los autores declaran que para esta investigación no se han realizado experimentos en seres humanos ni en animales.
Confidencialidad de los datos. Los autores declaran que han seguido los protocolos de su centro de trabajo sobre la publicación de datos de pacientes.

Derecho a la privacidad y consentimiento informado. Los autores declaran que en este artículo no aparecen datos de pacientes.

\section{Bibliografía}

1. Prats $P$, Ferrer $Q$, Rodríguez MA, Comas $C$. Prenatal diagnosis and outcome of congenital heart diseases. Diagnostico Prenat. 2011;22(4):128-35.

2. Sciacca P, Giacchi V, Mattia C, Greco F, Smilari P, Betta P, et al. Rhabdomyomas and Tuberous sclerosis complex: Our experience in 33 cases. BMC Cardiovasc Disord. 2014;14:1-11.

3. Anaya-Reyes $P$, Rodríguez-Rábago MJ. Diagnóstico prenatal de rabdomioma cardiaco. reporte de un caso. Ginecol Obstet Mex. 2013:81(8):477-81.

4. Bonnamy L, Perrotin F, Megier P, Haddad G, Body G, Lansac J. Fetal intracardiac tumor(s): Prenatal diagnosis and managementThree case reports. Eur J Obstet Gynecol Reprod Biol. 2001;99(1):112-7.

5. Gedikbasi A, Oztarhan K, Ulker V, Aslan G, Gul A, Sener-Arslan E, et al. Prenatal sonographic diagnosis of tuberous sclerosis complex. J Clin Ultrasound. 2011;39(7):427-30.

6. Groves AMM, Fagg NLK, Cook AC, Allan LD. Cardiac tumours in intrauterine life. Arch Dis Child. 1992;67(10 SPEC NO):1189-92.

7. Lacey SR, Donofrio MT. Fetal cardiac tumors: Prenatal diagnosis and outcome. Pediatr Cardiol. 2007;28(1):61-7.

8. D’Addario V, Pinto V, Di Naro E, Del Bianco A, Di Cagno L, Volpe P. Prenatal diagnosis and postnatal outcome of cardiac rhabdomyomas. J Perinat Med. 2002;30(2):170-5.

9. Hoadley SD, Wallace RL, Miller JF, Murgo JP. Prenatal diagnosis of multiple cardiac tumors presenting as an arrhythmia. J Clin Ultrasound. 1986;14(8):639-43.

10. Cavalcante CT de MB, Pinto Junior VC, Pompeu RG, Teles AC de O, Bandeira JA, Maia ICL, et al. Perinatal unusual rhabdomyoma location - case report and systematic reviews of the literature. J Matern Neonatal Med. 2019;7058:1-15.

11. Ekmekci E, Ozkan BO, Yildiz MS, Kocakaya B. Prenatal diagnosis of fetal cardiac rhabdomyoma associated with tuberous sclerosis: A case report. Case Reports Women's Heal. 2018;19:5-7.

12. Zhou QC, Fan P, Peng QH, Zhang M, Fu Z, Wang CH. Prenatal echocardiographic differential diagnosis of fetal cardiac tumors. Ultrasound Obstet Gynecol. 2004;23(2):165-71.

13. Shukla AK, Reddy AK, Latha A, Jayamohan AE. Cardiac rhabdomyoma: An antenatal illustration. BMJ Case Rep. 2015;2015:2014-5.

14. Brezinka C, Huter O, Haid C, Hammerer I, Dietze O. Prenatal diagnosis of a heart tumor. Am Heart J. 1988;116:563-6.

15. Choi JM, Jaffe R, Maidman J, Baxi LV. Multiple cardiac rhabdomyomas detected in utero. Fetal Diagn Ther. 2000;15(3):174-6.

16. Das BB, Sharma J. Cardiac rhabdomyoma and tuberous sclerosis: Prenatal diagnosis and follow-up. Indian J Pediatr. 2003;70(1):87-9.

17. Yuan SM. Fetal cardiac tumors: Clinical features, management and prognosis. J Perinat Med. 2018;46(2):115-21.

18. Pavlicek J, Klaskova E, Kapralova S, Prochazka M, Vrtel R, Gruszka T, et al. Fetal heart rhabdomyomatosis: a single-center experience. J Matern Neonatal Med. 2019;0(0):1-7. 